\title{
A BASIS OF THE GROUP OF PRIMITIVE ALMOST PYTHAGOREAN TRIPLES
}

\section{NIKOLAI A. KRYLOV}

Department of Mathematics

Siena College

515 Loudon Road

Loudonville NY 12211

USA

e-mail: nkrylov@siena.edu

\begin{abstract}
Let $m$ be a fixed square-free positive integer, then equivalence classes of solutions of Diophantine equation $x^{2}+m \cdot y^{2}=z^{2}$ form an infinitely generated abelian group under the operation induced by the complex multiplication. A basis of this group is constructed here using prime ideals and the ideal class group of the field $\mathbb{Q}(\sqrt{-m})$.
\end{abstract}

\section{Introduction}

Fix an arbitrary square-free integer $m>1$. Then the set of equivalence classes of solutions of Diophantine equation

$$
x^{2}+m \cdot y^{2}=z^{2},
$$

2010 Mathematics Subject Classification: 11R04, 11R11, $20 \mathrm{~F} 05$.

Keywords and phrases: almost Pythagorean triples, prime ideals, imaginary quadratic field, ideal class group.

Received September 20, 2016

(C) 2016 Scientific Advances Publishers 
will have a group structure. Indeed, given two arbitrary solutions $\left(a_{1}, b_{1}, c_{1}\right)$ and $\left(a_{2}, b_{2}, c_{2}\right)$ of (1) we can produce another one $(A, B, C)$ via the formulas

$$
A:=a_{1} a_{2}-m b_{1} b_{2}, \quad B:=a_{1} b_{2}+a_{2} b_{1}, \quad C:=c_{1} c_{2} .
$$

Here is precise definition of the group. Consider the set of all ordered primitive triples $(a, b, c) \in \mathbb{Z} \times \mathbb{Z} \times \mathbb{N}$ such that $a^{2}+m \cdot b^{2}=c^{2}$. A triple $(a, b, c)$ is called primitive when the $\operatorname{gcd}(a, b, c)=1$. Two triples $(a, b, c)$ and $(A, B, C)$ satisfying (1) are said to be equivalent if there exist $m, n \in \mathbb{Z} \backslash\{0\}$ such that $m(a, b, c)=n(A, B, C)$, where $m(a, b, c)$ $=(m a, m b,|m c|)$. This is clearly an equivalence relation. The equivalence class of $(a, b, c)$ will be denoted by $[a, b, c]$ and the set of all such classes will be denoted by $\mathcal{P}_{m}$ and called the group of primitive almost Pythagorean triples. Note that $[a, b, c]=[-a,-b, c]$ but $[a, b, c]$ $\neq[-a, b, c]$. Every equivalence class $[a, b, c] \in \mathcal{P}_{m}$ can be represented uniquely by a primitive triple $(\alpha, \beta, \gamma)$, where $\alpha>0$ and hence one could refer to primitive triples to describe elements of the group. Following the operation (2), we see that for any two classes $[a, b, c]$, $[A, B, C] \in \mathcal{P}_{m}$

$$
[a, b, c]+[A, B, C]=[a A-m b B, a B+b A, c C] .
$$

Here is an alternative description of this group using a bit more technical terms: Consider the multiplicative subgroup, say $\mathcal{A}_{m}$, of nonzero elements whose norm is a square of a rational number in the imaginary quadratic field $\mathbb{Q}(\sqrt{-m})$. The nonzero rational numbers $\mathbb{Q}^{*}$ will make a subgroup of $\mathcal{A}_{m}$ and it is easy to see that the corresponding factor group $\mathcal{A}_{m} / \mathbb{Q}^{*}$ is $\mathcal{P}_{m}$. 
This group has been considered by various authors (see, e.g., [1], [3], [4], and [5]) since the operation (2) was introduced by Taussky ([7]) for ordinary Pythagorean triples. It is well known that the group is infinitely generated and has torsion only when $m=3$. Description of a basis has been given however only for particular values of $m$ (see [1], [3], and [4]). The goal of this paper is to present a basis of the group $\mathcal{P}_{m}$ using prime ideals and the ideal class group of $\mathbb{Q}(\sqrt{-m})$, for all square-free integers $m>3$. For the proof of the following theorem, see [1] or [4].

Theorem. The group $\mathcal{P}_{m}$ is an infinitely generated abelian group for each square-free $m>1$. The identity element is $[1,0,1]$ and the inverse of $[a, b, c]$ is $[a,-b, c]=[-a, b, c] . \mathcal{P}_{m}$ has elements of finite order only when $m=3$.

Note that Zanardo and Zannier ([8]) studied the group consisting of the set of equivalence classes of solutions of $x^{2}+y^{2}=z^{2}$ in the ring $\mathcal{O}_{K}$ of integers of a number field $K$ and described a basis for the torsionfree part of that group. The group I consider here is a proper subgroup of the one discussed in [8].

From now on let's assume that we have a fixed, square-free integer $m>3$, and denote the imaginary quadratic field $\mathbb{Q}(\sqrt{-m})$ by $K$. The corresponding ring of integers will be denoted by $\mathcal{O}_{K}$ and the ideal class group of $K$ by $C l(K)$. The norm of a nonzero ideal $Q$ of $\mathcal{O}_{K}$ will be written as $N(Q)$. I will denote the set of all rational prime numbers by $\mathbb{P}$ and the set of all prime ideals of $\mathcal{O}_{K}$ by $P\left(\mathcal{O}_{K}\right)$, respectively.

\section{Ideal Classes of Order 2}

Lemma 1. Let $c=2^{i_{0}} \cdot p_{1}^{i_{1}} \cdot \ldots \cdot p_{k}^{i_{k}}$ be the prime decomposition of a natural number $c$. If $i_{0} \neq 0$, assume that $-m \equiv 1(\bmod 4)$, i.e., 2 is either inert or splits in $K$. Suppose that for each odd prime $p_{i}, i \in\{1,2, \ldots, k\}$ 
there exists a prime ideal $Q_{i}$ of $\mathcal{O}_{K}$ s.t. $N\left(Q_{i}\right)=p_{i}$, and let $Q_{0}=\langle 2\rangle$ if 2 is inert in $K$ and $Q_{0} \cdot Q_{0}^{\prime}=\langle 2\rangle$ if 2 splits in $K$ (here $Q_{0}^{\prime}$ is the conjugate of a prime ideal $\left.Q_{0}\right)$. If the class of $Q_{0}^{i_{0}} \cdot Q_{1}^{i_{1}} \cdot \ldots \cdot Q_{k}^{i_{k}}$ has order 2 in $C l(K)$, then there exist integers $u$ and $v$ s.t. either $u^{2}+m v^{2}=c^{2}$ or $u^{2}+m v^{2}=(2 c)^{2}$.

Proof. If $\left[Q_{0}^{i_{0}} \cdot Q_{1}^{i_{1}} \cdot \ldots \cdot Q_{k}^{i_{k}}\right]^{2}=[1]$, then there exists $z \in \mathcal{O}_{K}$ s.t.

$$
\prod_{t=0}^{k} Q_{t}^{2 i_{t}}=\langle z\rangle=\left\langle\frac{u+v \sqrt{-m}}{2^{1-\delta}}\right\rangle
$$

with $u, v \in \mathbb{Z} ; \delta=0$ if $-m \equiv 1(\bmod 4)$, and $\delta=1$ otherwise. If 2 splits or ramifies in $K$, we can take the norm of both ends of the above equality to obtain $4^{1-\delta} c^{2}=u^{2}+m v^{2}$. If 2 is inert in $K$, then we apply the previous step to the product of prime ideals $\left[Q_{1}^{i_{1}} \cdot \ldots \cdot Q_{k}^{i_{k}}\right]^{2}=[1]$ to obtain

$$
4\left(\frac{c}{2^{i_{0}}}\right)^{2}=u^{2}+m v^{2}, \quad \text { which implies }(2 c)^{2}=\left(2^{i_{0}} u\right)^{2}+m\left(2^{i_{0}} v\right)^{2} .
$$

Since every prime ideal of $\mathcal{O}_{K}$ lies over a unique rational prime, and every prime $p \in \mathbb{N}$ lies below at least one prime ideal of $\mathcal{O}_{K}$ (see Theorem 20 of [6]), we have the canonical projection $\mu: P\left(\mathcal{O}_{K}\right) \rightarrow \mathbb{P}$, which has a lifting map

$$
l: \mathbb{P} \rightarrow P\left(\mathcal{O}_{K}\right) \text { such that } \mu \circ l=I d_{\mathbb{P}} .
$$

Hence if we pick such a lifting $l$ and compose it with another canonical projection $\pi: P\left(\mathcal{O}_{K}\right) \rightarrow C l(K)$ we obtain a map $f$ and the following commutative diagram: 


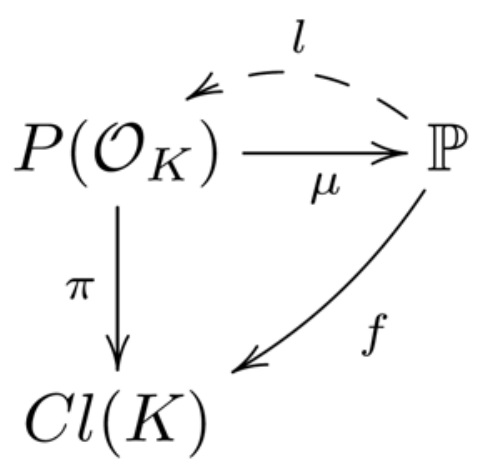

Note 1. Map $f$ does depend on the choice of the lifting $l: \mathbb{P} \rightarrow P\left(\mathcal{O}_{K}\right)$.

Note 2. Since $\pi$ is a multiplicative homomorphism from the group generated by $P\left(\mathcal{O}_{K}\right)$, we can extend such map $f$ in a natural way, to a multiplicative function from $\mathbb{N}$ to $C l(K)$.

Note 3. An odd prime $p$ divides $c$ for a primitive triple $(a, b, c)$, which satisfies (1), if and only if the Legendre symbol $\left(\frac{-m}{p}\right)=1$ (see Lemma 1.7 of [2]). It is also trivial to see that if $c$ is even in the primitive triple above, then we must have $-m \equiv 1(\bmod 4)$, and if $-m \equiv 5(\bmod 8)$, then $4 \nmid c$.

Let me now introduce two special subsets of $C l(K)$ and of $\mathbb{P}$, respectively.

Definition 1. The set of all elements of the ideal class group of $K$ which have order at most 2 , will be denoted by $E$, that is,

$$
E:=\left\{[A] \in C l(K) \mid A \text { is an ideal of } \mathcal{O}_{K} \text { and }[A]^{2}=[1]\right\} .
$$

Clearly, $E$ is a subgroup of $C l(K)$. 
Definition 2. A subset $L \subset \mathbb{P}$ is defined as the subset of rational primes that split (completely) in $K$. In the other words,

$$
L:=\left\{p \in \mathbb{P} \mid\left(\frac{-m}{p}\right)=1\right\},
$$

where $\left(\frac{-m}{p}\right)$ is the Legendre symbol for $p>2$ and the Kronecker symbol if $p=2$. Moreover, we define

$L_{0}:=f^{-1}(E) \cap L$, where $f^{-1}(E)$ denotes the preimage of $E$ in $\mathbb{P}$.

Claim 1. If $-m \equiv 1(\bmod 8)$ and $m>16$, then $2 \notin L_{0}$, and $E \subsetneq C l(K)$.

Proof. Since $-m \equiv 1(\bmod 8)$, we have the splitting of $\langle 2\rangle$ into the product of prime ideals

$$
\langle 2\rangle=\left\langle 2, \frac{1+\sqrt{-m}}{2}\right\rangle \cdot\left\langle 2, \frac{1-\sqrt{-m}}{2}\right\rangle=P \cdot P^{\prime},
$$

and it's enough to show that $P^{2}=\left\langle 4, \frac{1-m+2 \sqrt{-m}}{4}\right\rangle$ is not principal in $\mathcal{O}_{K}$. If one assumes that there are $x, y \in \mathbb{Z}$ s.t. $P^{2}=\left\langle\frac{x+y \sqrt{-m}}{2}\right\rangle$, then we could find $a, b \in \mathbb{Z}$ s.t.

$$
4=\frac{a+b \sqrt{-m}}{2} \cdot \frac{x+y \sqrt{-m}}{2}, \text { and hence } 16^{2}=\left(a^{2}+m b^{2}\right) \cdot\left(x^{2}+m y^{2}\right) .
$$

It's easy to see that assumptions like $b=0$ or $y=0$ lead to a contradiction (for example, by using the norm), and if by by $\neq 0$, then $\left(a^{2}+m b^{2}\right) \cdot\left(x^{2}+m y^{2}\right)>16^{2}$, which is also not allowed.

Note 4. If $m=7$, then $P^{2}=\left\langle\frac{-3+1 \sqrt{-7}}{2}\right\rangle$, and $[-3,1,4] \in \mathcal{P}_{7}$. If $m=15$, then $P^{2}=\left\langle\frac{1+1 \sqrt{-15}}{2}\right\rangle$, and $[1,1,4] \in \mathcal{P}_{15}$. 
Recall that Theorem 2 of [4] describes a basis of $\mathcal{P}_{m}$ for four special cases of $m$, which correspond to the class numbers $\{1,1,2,2\}$ of $\mathcal{O}_{K}$. It says:

Fix $m \in\{2,3,5,6\}$. Then $\mathcal{P}_{m}$ is generated by all primitive triples $(a, b, p)$, where $a>0$ and $p$ is prime such that there exist $u, v \in \mathbb{Z}$ with $p=u^{2}+m v^{2}$, or $2 p=u^{2}+m v^{2}$.

Our next theorem generalizes results of [4] and [1] (cf. also with [3]), and gives a basis of the group $\mathcal{P}_{m}$ when every element of the ideal class group $C l(K)$ has order at most 2. Approach of Baldisserri in [1] covered several particular cases of $m$ (see Theorem 2 on page 305 of [1]). In all those 36 cases, $C l(K)$ is either trivial or isomorphic to the direct product of several copies (up to 4 ) of $\mathbb{Z}_{2}$ (the cyclic group of order 2 ).

Theorem 1. If $L_{0}=L$, i.e., the ideal class group $C l(K)$ has exponent less than or equal to 2 , then the group $\mathcal{P}_{m}$, with $m>3$, is generated freely by the set of all primitive triples $(a, b, p)$ or $(a, b, 2 p)$, where $p \in L$ and $a$ and $b$ are positive integers such that $a^{2}+m b^{2}=p^{2}$ or $a^{2}+m b^{2}=(2 p)^{2}$.

Proof. First let's prove that if $p \in L_{0}$, then there exists a unique coprime pair $(a, b) \in \mathbb{N} \times \mathbb{N}$ s.t. either $a^{2}+m b^{2}=p^{2}$ or $a^{2}+m b^{2}=(2 p)^{2}$, but not both. If $p \in L_{0}$, then from our Lemma 1 , we deduce that there exist integers $u$ and $v$ s.t. either $u^{2}+m v^{2}=p^{2}$ or $u^{2}+m v^{2}=(2 p)^{2}$. If we assume that $p$ is odd and there are two pairs of relatively prime integers $(a, b)$ and $(u, v)$ s.t. $a^{2}+m b^{2}=p^{2}$ and at the same time $u^{2}+m v^{2}=(2 p)^{2}$, we consider two new elements of $\mathcal{P}_{m}$ :

$$
[u, v, 2 p] \pm[a, b, p]=\left\{\begin{array}{l}
{\left[a u-m b v, a v+b u, 2 p^{2}\right]} \\
{\left[-a u-m b v,-a v+b u, 2 p^{2}\right]}
\end{array}\right.
$$


Since $(b u+a v)(b u-a v)=b^{2} u^{2}+b^{2} m v^{2}-\left(a^{2} v^{2}+m b^{2} v^{2}\right)=p^{2}\left(4 b^{2}-v^{2}\right)$, if $v \neq \pm 2 b$ we have $p \mid b u+a v$ or $p \mid b u-a v$ or both. In the last case we'd have $p \mid 2 b u$, which is impossible for an odd prime and two pairs of relatively prime integers $(a, b)$ and $(u, v)$. If $p$ divides only $b u+a v$, then $p^{2} \mid b u+a v$, and hence $p^{2} \mid a u-m b v$, i.e., $[u, v, 2 p]+[a, b, p]=[s, t, 2]$ for some integers $s$ and $t$. Since $m>3$ we deduce that $t=0$ and $a v=-b u$, which contradicts the assumptions. If $p$ divides only $b u-a v$, a similar argument leads to the contradiction as well. If $v= \pm 2 b$, we again have a contradiction with $(u, v)=1$. Analogously, one can consider the remaining cases when $p$ is odd and prove the uniqueness of a coprime pair $(a, b) \in \mathbb{N} \times \mathbb{N}$ s.t. either $a^{2}+m b^{2}=p^{2}$ or $a^{2}+m b^{2}=(2 p)^{2}$. If $p=2$, we could have $a^{2}+m b^{2}=16$ only for $[3,1,4] \in \mathcal{P}_{7}$ or for $[1,1,4] \in \mathcal{P}_{15}$.

Secondly, we prove that the set of all such primitive triples $\left(a, b, 2^{\tau} p\right)$, with $p \in L_{0}$ and $\tau \in\{0,1\}$ generate the group $\mathcal{P}_{m}$. Take any primitive $[a, b, c] \in \mathcal{P}_{m}$ and assume that the prime decomposition of $c$ is $c=2^{i_{0}} \cdot p_{1}^{i_{1}} \cdot \ldots \cdot p_{k}^{i_{k}}$, where $i_{0} \geq 0$ and $p_{i}$ are distinct odd rational primes. Then by Note 3 and our Lemma 1 , for each $i \in\{1, \ldots, k\}$ there exist $u_{i}, v_{i} \in \mathbb{N} \times \mathbb{N}$ s.t. $u_{i}^{2}+m v_{i}^{2}=p_{i}^{2}$ or $u_{i}^{2}+m v_{i}^{2}=\left(2 p_{i}\right)^{2}$, that is, either $\left[u_{i}, v_{i}, p_{i}\right]$ or $\left[u_{i}, v_{i}, 2 p_{i}\right]$ is a generator. Assume without loss of generality that $i_{1}>0$ and consider

$$
[a, b, c] \pm\left[u_{1}, v_{1}, 2^{\tau} \cdot p_{1}\right]=\left\{\begin{array}{l}
{\left[a u_{1}-m b v_{1}, a v_{1}+b u_{1}, c \cdot 2^{\tau} \cdot p_{1}\right]} \\
{\left[-a u_{1}-m b v_{1},-a v_{1}+b u_{1}, c \cdot 2^{\tau} \cdot p_{1}\right]}
\end{array}\right.
$$

with $\tau \in\{0,1\}$. As above, we have $\left(b u_{1}+a v_{1}\right)\left(b u_{1}-a v_{1}\right) \equiv 0\left(\bmod p_{1}^{2}\right)$, and hence $p_{1}^{2}$ divides at least one of $\left(b u_{1}+a v_{1}\right)$ or $\left(b u_{1}-a v_{1}\right)$, or $p_{1}$ divides each of these numbers. Assuming the last possibility we'll get a 
contradiction with the fact that our triples on the left hand side of (3) are primitive. Assuming the first possibility we obtain that $p_{1}^{2} \mid a u_{1}-m b v_{1}$. Then we have for $D=\left(a u_{1}-m b v_{1}\right) / p_{1}^{2}$ and $E=\left(a v_{1}+b u_{1}\right) / p_{1}^{2}$,

$$
[a, b, c]=\left[-u_{1}, v_{1}, 2^{\tau} \cdot p_{1}\right]+\left[D, E, 2^{\tau} \cdot\left(\frac{c}{p_{1}}\right)\right],
$$

which implies that there exist integers $\gamma$ and $\omega$ such that

$$
[a, b, c]=\sum_{j=1}^{k} \pm r_{j} \cdot\left[u_{j}, v_{j}, 2^{\tau_{j}} p_{j}\right]+\left[\gamma, \omega, 2^{n}\right]
$$

where $\tau_{j} \in\{0,1\}$ and $r_{j} \in\left\{0, \ldots, i_{j}\right\}$ for each $j \in\{1, \ldots, k\}$, and $n \geq 0$. According to our Note $3,\left[\gamma, \omega, 2^{n}\right]=[1,0,1]$, unless $-m \equiv 1(\bmod 8)$. In the latter case, Claim 1 guaranties that $m \in\{7,15\}$, and one can easily prove using induction and applying the divisibility argument we just used above to the elements

$$
\left[\gamma, \omega, 2^{n}\right] \pm[q, r, 4]=\left\{\begin{array}{l}
{\left[q \gamma-m r \omega, q \omega+r \gamma, 2^{n+2}\right]} \\
{\left[-q \gamma-m r \omega,-q \omega+r \gamma, 2^{n+2}\right],}
\end{array}\right.
$$

that $\left[\gamma, \omega, 2^{n}\right]= \pm(n-1) \cdot[q, r, 4]$, where $[q, r, 4]=[3,1,4]$ if $m=7$, and $[q, r, 4]=[1,1,4]$ if $m=15$, respectively.

Thirdly, we show that the generating set is free of any nontrivial relations. Suppose that there exists a primitive triple $[K, L, M] \in \mathcal{P}_{m}$ with two different presentations by elements from the generating set, i.e.,

$$
\sum_{j \in J} z_{j}=[K, L, M]=\sum_{t \in T} z_{t}
$$

where each $z_{i}=\left[a_{i}, b_{i}, 2^{\tau} p_{i}\right]$, with $\tau \in\{0,1\}$ and $i \in J \cup T$ is a primitive triple with $p_{i} \in L_{0}$. We can assume without loss of generality that $J \cap T=\emptyset$. Since the third components get multiplied when we add 
two elements of $\mathcal{P}_{m}$, we can write the third components of the left and right hand sides of (4) (before reducing to the corresponding primitive triples) as

$$
2^{j_{0}} \cdot \prod_{j \in J} p_{j} \quad \text { and } \quad 2^{t_{0}} \cdot \prod_{t \in T} p_{t}, \quad \text { respectively. }
$$

Since the left and right hand sides of (4) are equal, and since $J \cap T=\emptyset$, we see that $M=2^{n}, n \geq 0$. Then we deduce from our Note 3 above that $[K, L, M]=[1,0,1]$, unless $-m \equiv 1(\bmod 8)$. Suppose now that $-m \equiv 1$ $(\bmod 8)$, and the third component of one of $z_{j}$ is divisible by an odd prime $p$. Then we could write the left hand side of (4) as $k \cdot\left[a, b, 2^{\tau} \cdot p\right]$ $+[U, V, W]$, where $k \geq 1$ and $\tau \in\{0,1\}$. Writing $k \cdot\left[a, b, 2^{\tau} \cdot p\right]$ as $\left[\alpha, \beta, 2^{\varepsilon} \cdot p^{k}\right]$ (see Lemma 2 below), we can rewrite the left hand side of (4) as $\left[\alpha, \beta, 2^{\varepsilon} \cdot p^{k}\right]+[U, V, W]$, where the triples $\left(\alpha, \beta, 2^{\varepsilon} \cdot p^{k}\right)$ and $(U, V, W)$ are not necessarily primitive, but $(p, W)=1$ and $(\beta, p)=1$. Thus

$\left[\alpha, \beta, 2^{\varepsilon} \cdot p^{k}\right]+[U, V, W]=\left[\alpha U-m \beta V, \alpha V+\beta U, 2^{\varepsilon} \cdot p^{k} \cdot W\right]=\left[K, L, 2^{n}\right]$, which implies that $p \mid \alpha U-m \beta V$, and also $p \mid \alpha V+\beta U$. In such situation we would also have $p \mid W$, which is not allowed. Thus the left and right hand sides of (4) could only have group elements where the third component is a power of 2 . But there is only one such basis element, which is $[*, *, 4]$, and hence we can not have two different nontrivial presentations of $[K, L, M]$.

Lemma 2. Suppose for an odd prime $p$ we have a primitive triple $\left[u, v, p^{b} w\right] \in \mathcal{P}_{m} \quad$ s.t. $\quad(p, w)=1$, and $b \geq 1$. Take any $n \in \mathbb{N}$. If $\left[u_{n}, v_{n}, w_{n}\right]=n \cdot\left[u, v, p^{b} w\right]$ with $\left(u_{n}, v_{n}\right)=1$, then $p^{n b} \mid w_{n}$. 
Proof. First observe that $\left[u_{2}, v_{2}, w_{2}\right]=\left[u^{2}-m v^{2}, 2 u v, p^{2 b} w^{2}\right]$ and if $p^{2 b}$ does not divide $w_{2}$ we must have $p \mid 2 u v$, which is not allowed. Let us write $u^{2}-m v^{2}=\left(p^{b} w\right)^{2}-2 m v^{2}=p \cdot A_{2}-2 m v^{2}$ for some $A_{2} \in \mathbb{Z}$. Then one can use induction to show that

$$
\begin{aligned}
& {\left[u_{2 k}, v_{2 k}, w_{2 k}\right]=\left[p \cdot A_{2 k} \pm 2^{2 k-1} \cdot m^{k} \cdot v^{2 k},\right.} \\
& \left.\quad p \cdot B_{2 k}- \pm 2^{2 k-1} \cdot m^{k-1} \cdot u \cdot v^{2 k-1}, p^{2 k b} \cdot w^{2 k}\right],
\end{aligned}
$$

with both $A_{2 k}$ and $B_{2 k} \in \mathbb{Z}$. Analogously one proves a similar formula when $n$ is odd, where the second component of $(2 k+1) \cdot\left[u, v, p^{b} w\right]$ will be $v_{2 k+1}=p \cdot B_{2 k+1} \pm 2^{2 k} \cdot m^{k} \cdot v^{2 k+1}$. Clearly, in both cases these formulas imply that $p^{n b} \mid w_{n}$.

Example 1. Let $m=35$, then $C l(K) \cong C_{2}$ and we have $L_{0}=L=\{3,11,13,17,29,47,71,73,79,83,97,103,109,149,151, \ldots\}$.

Here are first few generating triples which satisfy equation $a^{2}+m b^{2}=p^{2}$ :

$$
\{[1,12,71],[17,12,73],[43,12,83],[131,12,149], \ldots\},
$$

and here are several first generating triples satisfying equation $a^{2}+m b^{2}=(2 p)^{2}:$

$\{[1,1,2 \cdot 3],[13,3,2 \cdot 11],[19,3,2 \cdot 13],[29,3,2 \cdot 17],[23,9,2 \cdot 29]$,

$$
[31,15,2 \cdot 47],[157,3,2 \cdot 79], \ldots\} \text {. }
$$

\section{Ideal Classes of Higher Order}

From now on I will assume that $C l(K)$ has elements of order higher than 2 and hence $E$ will be a proper subgroup of $C l(K)$. In such a case, we can extend the diagram defining map $f$ to the following one: 


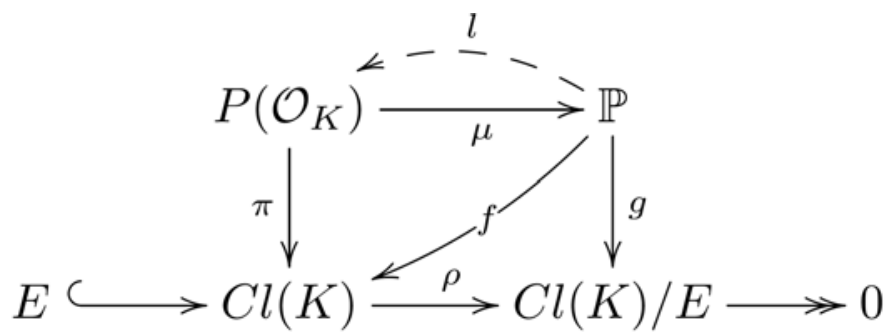

Here map $\rho$ is the canonical projection onto the factor group $C l(K) / E$, and the map $g:=\rho \circ f$.

Since $C l(K)$ is finite, we can present the factor group $C l(K) / E$ as a direct sum of cyclic groups $C l(K) / E \cong G_{1} \times G_{2} \times \cdots \times G_{n}$ with corresponding orders denoted by $h_{j}:=\left|G_{j}\right|, j \in\{1,2, \ldots, n\}$. For each $G_{j}$ pick an ideal $P_{j} \in P\left(\mathcal{O}_{K}\right)$, s.t. $G_{j}=\left\langle\rho \circ \pi\left(P_{j}\right)\right\rangle$, (c.f. with the presentation of the factor group $C l(S) / M$ as a direct sum of cyclic groups and the corresponding construction right after that on page 84 of [8]).

Now let's use the lifting $l: \mathbb{P} \rightarrow P\left(\mathcal{O}_{K}\right)$ to construct a map $\beta: L \rightarrow \mathcal{P}_{m}$ as follows. First, take any prime $p \in L \backslash L_{0}$.

Case 1. Suppose that $\mu\left(P_{j}\right)=p$ for some $j \in\{1,2, \ldots, n\}$. Then $\left(\pi\left(P_{j}\right)\right)^{h_{j}} \in E$ and by Lemma 1 above, we can write $\left(2^{1-\delta} p^{h_{j}}\right)^{2}=u^{2}+m v^{2}$ for some positive integers $u$ and $v$ (where as above, $\delta \in\{0,1\}$ ). If we suppose that $p \mid \operatorname{gcd}(u, v)$ we will deduce that $P_{j}^{\prime}$ (the conjugate ideal of $\left.P_{j}\right)$ divides in $\mathcal{O}_{K}$ a power of $P_{j}$, which is impossible, therefore we can assume that $\operatorname{gcd}(u, v)=1$. Thus, we obtained a primitive almost Pythagorean triple $\left(u, v, 2^{1-\delta} \cdot p^{h_{j}}\right)$, which is unique up to signs of $u$ and $v$. Therefore, we define

$$
\beta(p):=\left[u, v, 2^{1-\delta} \cdot p^{h_{j}}\right], \quad \text { where } \quad(u, v) \in \mathbb{N} \times \mathbb{N}
$$


Case 2. Suppose further that $p \in L \backslash\left(L_{0} \cup\left\{\mu\left(P_{j}\right) \mid j \in\{1,2, \ldots, n\}\right\}\right)$. Then for the prime ideal $P=l(p)$, there exist nonnegative integers $a_{j p} \in\left\{0,1, \ldots, h_{j}-1\right\}$ such that

$$
P \cdot P_{1}^{a_{1 p}} \cdot \ldots \cdot P_{n}^{a_{n p}} \in E \text {, and hence } P^{2} \cdot P_{1}^{2 a_{1 p}} \cdot \ldots \cdot P_{n}^{2 a_{n p}}=\left\langle\frac{u+v \sqrt{-m}}{2^{1-\delta}}\right\rangle,
$$

for some $(u, v) \in \mathbb{Z} \times \mathbb{Z}$.

\section{Claim 2.}

$$
\operatorname{gcd}\left(\frac{u}{2^{1-\delta}}, \frac{v}{2^{1-\delta}}\right)=1
$$

Proof. Suppose that we have a prime $q$, which splits and divides both $u$ and $v$. Then if $Q:=l(q)$, we have $\langle q\rangle=Q \cdot Q^{\prime} \mid P^{2} \cdot P_{1}^{2 a_{1 p}} \cdot \ldots \cdot P_{n}^{2 a_{n p}}$. But this is impossible since none of the ideals $\left\{P, P_{1}, \ldots, P_{n}\right\}$ has order 2 in $C l(K)$ and they all have different norms, while $N(Q)=N\left(Q^{\prime}\right)=q$.

Thus, as in the first case, we found a relatively prime pair of integers $(u, v)$ s.t.

$$
u^{2}+m v^{2}=\left(2^{1-\delta} \cdot p \cdot p_{1}^{\alpha_{1 p}} \cdot \ldots \cdot p_{n}^{\alpha_{n p}}\right)^{2}
$$

Note that this time, the pair $(u, v)$ is not necessarily unique. If, for example, $h_{1}=2 t$, and we found a triple $\left(u_{1}, v_{1}, 2^{1-\delta} \cdot p \cdot p_{1}^{t}\right)$, then for the triple $\left(u, v, 2^{1-\delta} \cdot p^{h_{1}}\right)$ found in Case 1 above, one of the triples

$$
\left[u_{1}, v_{1}, 2^{1-\delta} \cdot p \cdot p_{1}^{t}\right] \pm\left[u, v, 2^{1-\delta} \cdot p^{h_{1}}\right]
$$

would be another primitive almost Pythagorean triple with the third component $2^{1-\delta} \cdot p \cdot p_{1}^{t}$ and the first two components different from $\pm u_{1}$ and $\pm v_{1}$. We can alternate the construction giving (6) and show that each 
$a_{j p}$ may be taken from the set $\left\{0,1, \ldots,\left[\frac{h_{j}}{2}\right]\right\}$. Indeed if, for example, $a_{1 p}>\left[\frac{h_{1}}{2}\right]$, we can multiply the product $P \cdot P_{1}^{a_{1 p}} \cdot \ldots \cdot P_{n}^{a_{n p}}$ by $\left\langle p_{1}\right\rangle^{h_{1}-a_{1 p}}=\left(P_{1} \cdot P_{1}^{\prime}\right)^{h_{1}-a_{1 p}}$ and obtain an element

$$
P \cdot\left(P_{1}^{\prime}\right)^{b_{1 p}} \cdot \ldots \cdot P_{n}^{a_{n p}} \in E \text { with } b_{1 p}=h_{1}-a_{1 p} \leq\left[\frac{h_{1}}{2}\right] .
$$

Now we are ready to define map $\beta: L \backslash L_{0} \rightarrow \mathcal{P}_{m}$ for $p \in L \backslash\left(L_{0} \cup\right.$ $\left.\left\{\mu\left(P_{j}\right) \mid j \in\{1,2, \ldots, n\}\right\}\right)$. Take any such prime number $p$, then as we just explained above, there exist relatively prime integers $u$ and $v$ such that $u^{2}+m v^{2}=\left(2^{1-\delta} \cdot p \cdot p_{1}^{a_{1 p}} \cdot \ldots \cdot p_{n}^{a_{n p}}\right)^{2}$, and $\left\{a_{1 p}, \ldots, a_{n p}\right\}$ are the coordinates of the ideal $P=l(p)$ in $C l(K) / E$ written in the basis $\left\{\rho \circ \pi\left(P_{1}\right)\right.$ or $\rho \circ \pi\left(P_{1}^{\prime}\right), \ldots, \rho \circ \pi\left(P_{n}\right)$ or $\left.\rho \circ \pi\left(P_{n}^{\prime}\right)\right\}$, which means that $a_{j p} \leq\left[\frac{h_{j}}{2}\right]$ for each $j$. Among such primitive triples $\left(u, v, 2^{1-\delta} \cdot p \cdot p_{1}^{a_{1 p}}\right.$ $\cdot \ldots \cdot p_{n}^{a_{n p}}$ ) take the one with the smallest value of $|u|$, say $u_{0}$, and define

$$
\beta(p):=\left[u_{0}, v_{0}, 2^{1-\delta} \cdot p \cdot p_{1}^{a_{1 p}} \cdot \ldots \cdot p_{n}^{a_{n p}}\right], \text { where }\left(u_{0}, v_{0}\right) \in \mathbb{N} \times \mathbb{N}
$$

We can extend map $\beta$ to elements of $L_{0}$ as well, by defining $\beta(p)$ := $[a, b, p]$ if $a^{2}+m b^{2}=p^{2}$, and $\beta(p):=[a, b, 2 p]$ if $a^{2}+m b^{2}=(2 p)^{2}$, when $p \in L_{0}$. Thus we obtain a map $\beta: L \rightarrow \mathcal{P}_{m}$.

Note 5. It is clear from the construction that this map $\beta: L \rightarrow \mathcal{P}_{m}$ is one-to-one. It is also clear that $\beta$ depends on several choices we've made in the construction, and in particular it depends on the choice of the prime ideals projecting onto the generators of subgroups $G_{j}$ of $C l(K) / E$. 
Example 2. Let $m=23$, then $C l(K) \cong C_{3}$ and we have

$L=\{2,3,13,29,31,41,47,59,71,73,101,127,131,139,151,163,167$, $173,179,193,197, \ldots\}$.

Here $L_{0}=\{59,101,167,173, \ldots\} \subset L$ with the corresponding values $\beta(59)=[13,12,59], \beta(101)=[83,12,101], \beta(167)=[121,24,167]$, $\beta(173)=[11,36,173], \ldots$

If we choose the ideal $\left\langle 2, \frac{1+\sqrt{-23}}{2}\right\rangle$ lying over 2 as the one, which gives the generator $P_{1}$ of $C l(K) / E=C_{3}$, then $\beta(2)=\left[7,3,2 \cdot 2^{3}\right]$, and also $\beta(3)=[11,1,2 \cdot(3 \cdot 2)], \beta(13)=[29,9,2 \cdot(13 \cdot 2)], \beta(29)=[91,15,2 \cdot(29 \cdot 2)], \ldots$ and so on ...

If we choose the ideal $\langle 3,1+\sqrt{-23}\rangle$ lying over 3 as the one, which gives the generator of $C l(K) / E=C_{3}$, then $\beta(3)=\left[19,4,3^{3}\right]$, and also $\beta(2)=[11,1,2 \cdot(2 \cdot 3)], \beta(13)=[7,8,(13 \cdot 3)], \beta(29)=[41,16,(29 \cdot 3)], \ldots$ and so on ....

Using this map $\beta$, we can now describe a basis of the group $\mathcal{P}_{m}$ for all $m>3$ when the corresponding ideal class group $C l(K)$ has elements or order higher than 2 (cf. with Theorem 3 of [8]).

Theorem 2. $\operatorname{Im}(\beta) \subset \mathcal{P}_{m}$ forms a basis of the free abelian group $\mathcal{P}_{m}$, $m>3$.

Proof. First I explain why $\operatorname{Im}(\beta)$ is a generating set. Take any primitive $[a, b, c] \in \mathcal{P}_{m}$ and write the prime decomposition in $\mathcal{O}_{K}$ for the ideal

$\langle a-b \sqrt{-m}\rangle=T_{1}^{l_{1}} \cdot T_{2}^{l_{2}} \cdot \ldots \cdot T_{r}^{l_{r}}=: \mathcal{T}$, and note that $2 \mid l_{j} \forall_{j}$, since $(a, b)=1$. 
If $T_{1}$ is not one of the chosen generators $\left\{P_{1}, P_{2}, \ldots, P_{n}\right\}$ for $C l(K) / E$, then using the construction we used in Case 2, we can write that $T_{1} \doteq Q_{1}^{b_{1 t_{1}}} \cdot Q_{2}^{b_{2 t_{1}}} \cdot \ldots \cdot Q_{n}^{b_{n t_{1}}}$, where $\doteq$ means the equality up to a product by an element of $E, t_{1}=N\left(T_{1}\right)$ and each $Q_{j} \in\left\{P_{j}, P_{j}^{\prime}\right\}$ so that $b_{j t_{1}} \in\left\{0,1, \ldots,\left[\frac{h_{j}}{2}\right]\right\}$. Denote the product $T_{1}^{\prime} \cdot Q_{1}^{b_{1 t_{1}}} \cdot Q_{2}^{b_{2 t_{1}}} \cdot \ldots \cdot Q_{n}^{b_{n t_{1}}}$ by $\mathcal{T}_{1}$ and we obtain

$$
\mathcal{T} \cdot \mathcal{T}_{1}^{l_{1}} \doteq T_{2}^{l_{2}} \cdot \ldots \cdot T_{r}^{l_{r}} \cdot\left(Q_{1}^{b_{1 t_{1}}} \cdot Q_{2}^{b_{2 t_{1}}} \cdot \ldots \cdot Q_{n}^{b_{n t_{1}}}\right)^{l_{1}}
$$

Similarly we can "eliminate" from $\mathcal{T}$ all $T_{j}$, which are not in the set $\left\{P_{1}, P_{2}, \ldots, P_{n}\right\}$. Suppose that on the other hand we have $T_{1}=P_{1}$ (we can always rename $T_{j}$ if needed). Then using division with remainder we can write $l_{1}=q_{1} \cdot h_{1}+r_{1}$, with $0 \leq r_{1}<h_{1}$ and hence $\left(T_{1}^{\prime}\right)^{l_{1}}=\mathcal{T}_{1}^{q_{1}} \cdot Q_{1}^{r_{1}}$, where $Q_{1}=P_{1}^{\prime}$, and $\mathcal{T}_{1}=\left(P_{1}^{\prime}\right)^{h_{1}}$, which also would give us

$$
\mathcal{T} \cdot \mathcal{T}_{1}^{q_{1}} \doteq T_{2}^{l_{2}} \cdot \ldots \cdot T_{r}^{l_{r}} \cdot\left(Q_{1}^{\prime}\right)^{r_{1}}
$$

Therefore, if we let $\omega_{j}=q_{j}$ when $T_{j}=P_{j}$ and $l_{j}=q_{j} \cdot h_{j}+r_{j}$, and $\omega_{j}=l_{j}$ otherwise, we deduce that

$$
\mathcal{T} \cdot \mathcal{T}_{1}^{\omega_{1}} \cdot \ldots \cdot \mathcal{T}_{r}^{\omega_{r}} \in E
$$

since each $\mathcal{T}_{j} \in E$ and $\mathcal{T}$ is principal. Hence we can write for the conjugate ideal

$$
\langle a+b \sqrt{-m}\rangle=\mathcal{T}_{1}^{\omega_{1}} \cdot \ldots \cdot \mathcal{T}_{r}^{\omega_{r}} \cdot I, \text { for some } I \in E .
$$

Since each $l_{j}$ is even, this last equality implies

$$
[a, b, c]=\gamma_{1} \beta\left(t_{1}\right)+\gamma_{2} \beta\left(t_{2}\right)+\ldots+\gamma_{r} \beta\left(t_{r}\right)+[g, h, d], \text { with all } \gamma_{j} \in \mathbb{Z},
$$

and where $d$ can be divided only by 2 , primes from the set $L_{0}$, and powers of $\left(p_{j}\right)^{h_{j}}$ for $j \in\{1,2, \ldots, n\}$ (recall that by our choice, $C l(K) / E$ 
is generated by $\rho \circ \pi\left(P_{j}\right)$, where $\mu\left(P_{j}\right)=p_{j}$ and $h_{j}$ is the order of the corresponding cyclic subgroup $G_{j}$ ). Repeating the second step from the proof of Theorem 1 above, and presenting $[g, h, d]$ as a linear combination of elements from $\beta\left(L_{0}\right)$, and from $\left\{\beta\left(p_{1}\right), \ldots \beta\left(p_{n}\right)\right\}$ we conclude that $\mathcal{P}_{m}$ is generated by the image $\operatorname{Im}(\beta)$.

To show that the set $\operatorname{Im}(\beta)$ is free of any nontrivial relations I will use the same approach that was used in the third part of the proof of Theorem 1. Let's suppose that there is a nontrivial relation among the generators, i.e., there exists a primitive triple $(K, L, M)$ s.t. $[K, L, M] \in \mathcal{P}_{m}$ and

$$
\sum_{i \in I} s_{i} \cdot \beta\left(p_{i}\right)=[K, L, M]=\sum_{j \in J} t_{j} \cdot \beta\left(p_{j}\right), \text { and } I \cap J=\emptyset,
$$

where $\left\{p_{i} \mid i \in I\right\} \cup\left\{p_{j} \mid j \in J\right\} \subset L$, and $s_{i}, t_{j} \in \mathbb{Z}$ for all $i \in I$ and $j \in J$. If we assume that there exists an odd prime $q \in\left\{p_{i} \mid i \in I\right\}$, which does not lie below any of the chosen idelas $P_{1}, \ldots, P_{n}$ mapping into the generators of $C l(K) / E$, then using our Lemma 2, we can write the left hand side of (7) as

$$
\sum_{i \in I, p_{i} \neq q} s_{i} \cdot \beta\left(p_{i}\right)+s \cdot \beta(q)=\left[K_{1}, L_{1}, M_{1}\right]+\left[u, v, q^{s} \cdot w\right]
$$

where the triples $\left(K_{1}, L_{1}, M_{1}\right)$ and $\left(u, v, q^{s} \cdot w\right)$ are primitive. It follows from the definition of operation in $\mathcal{P}_{m}$, fact that $I \cap J=\emptyset$, and our construction of map $\beta$ that $\left(M_{1}, q\right)=1$ and also $(M, q)=1$. But

$[K, L, M]=\left[K_{1}, L_{1}, M_{1}\right]+\left[u, v, q^{s} \cdot w\right]=\left[u K_{1}-m v L_{1}, v K_{1}+u L_{1}, q^{s} \cdot w \cdot M_{1}\right]$,

which is possible only when $q^{s}$ divides both $u K_{1}-m v L_{1}$, and $v K_{1}+u L_{1}$. In such case $q^{s}$ will also divide $v K_{1}^{2}+m v L_{1}^{2}=v \cdot M_{1}^{2}$, which is possible only if $s=0$. This proves that if a nontrivial relation exists among elements of $\operatorname{Im}(\beta)$, it could only involve elements from $\beta\left(L_{0}\right)$ and from $\left\{\beta(2), \beta\left(p_{1}\right), \ldots, \beta\left(p_{n}\right)\right\}$. 
If we assume that there is a nontrivial relation (7), with $\left\{p_{i} \mid i \in I\right\}$ $\bigcup\left\{p_{j} \mid j \in J\right\} \subset L_{0} \cup\left\{p_{1}, \ldots, p_{n}\right\}, \quad$ and, for example, that odd $p_{1} \in\left\{p_{i} \mid i \in I\right\}$, then using the definition of map $\beta$ and Lemma 2, we can rewrite the left hand side of (7) this time as

$$
\sum_{i \in I, p_{i} \neq p_{1}} s_{i} \cdot \beta\left(p_{i}\right)+s_{1} \cdot \beta\left(p_{1}\right)=\left[K_{1}, L_{1}, M_{1}\right]+\left[u, v, p_{1}^{s_{1} h_{1}} \cdot w\right]
$$

where $\left(K_{1}, L_{1}, M_{1}\right)$ and $\left(u, v, p_{1}^{s_{1}} h_{1} \cdot w\right)$ are primitive triples such that $\left(M_{1}, p_{1}\right)=1$. Since $p_{1} \notin\left\{p_{j} \mid j \in J\right\}$, we also have $\left(M, p_{1}\right)=1$. We will come to a contradiction again using exactly the same argument we just used above by presenting $[K, L, M]$ as $\left[*, *, p_{1}^{s_{1} h_{1}} \cdot w \cdot M_{1}\right]$. Hence the elements from $\left\{\beta\left(p_{1}\right), \ldots, \beta\left(p_{n}\right)\right\}$ can not be involved in a nontrivial relation either (with only possible exception of $\beta(2)$ ).

Finally, assuming that there is a nontrivial relation among elements of $\left\{\beta(p) \mid p \in L_{0} \cup\{2\}\right\}$ only, one can repeat the third part of the proof of Theorem 1 to deduce that the left and right hand sides of (7) could only have group elements where the third component is a power of 2 . But there could be only one such basis element, which is $\beta(2)$, and hence we can not have two different nontrivial presentations of $[K, L, M]$. This finishes the proof of Theorem 2 .

Here is an example, which motivates and illustrates the approach I used above in the construction of map $\beta$.

Example 3. Let $m=974$, then $C l(K) \cong C_{12} \times C_{3}$. Choose the ideal $\langle 5,1+\sqrt{-m}\rangle$ as the generator $P_{1}$ of $C_{12}$ and the ideal $\langle 41,16+\sqrt{-m}\rangle$ as the generator $P_{2}$ of $C_{3}$. Then $\rho \circ \pi\left(P_{1}\right)$ and $\rho \circ \pi\left(P_{2}\right)$ will be the chosen generators of the factor group $C l(K) / E \cong C_{6} \times C_{3}$. Using a computer, one can easily find that subset $L_{0}$ starts with numbers 
$L_{0}=\{937,983, \ldots\}, \quad$ and correspondingly $\beta(937)=[37,30,937]$ and $\beta(983)=[965,6,983]$. On the other hand, the set $L$ contains many smaller primes as well:

$L=\{3,5,11,13,31,37,41,43,59,71,73,89,97,101,103,109,127,131$, $137,149,163, \ldots\}$.

For the ideal $P_{2}$, we have $P_{2}^{6}=\langle 61129-1020 \sqrt{-974}\rangle$ and $\beta(41)=\left[61129,1020,41^{3}\right]$ with $61129^{2}+974 \cdot 1020^{2}=\left(41^{3}\right)^{2}$. For the ideal $P_{1}$, we have $P_{1}^{12}=\langle 14651-174 \sqrt{-974}\rangle$ and $\beta(5)=\left[14651,174,5^{6}\right]$.

Now let's choose a prime which is not in $L_{0} \cup\left\{\mu\left(P_{1}\right), \mu\left(P_{2}\right)\right\}$, for example $p=3$. Then we have the product $P \cdot P_{1} \cdot P_{2} \in E$ since

$$
\langle 3,1+\sqrt{-974}\rangle \cdot\langle 5,1+\sqrt{-m}\rangle \cdot\langle 41,16+\sqrt{-m}\rangle=\langle 615,16+\sqrt{-974}\rangle,
$$

and $\langle 615,16+\sqrt{-974}\rangle^{2}=\langle 359-16 \sqrt{-974}\rangle$. Hence $\beta(3)=[359,16,3$. $5 \cdot 41]$.

Notice also that we have two primitive triples for $p=37$ where the third components are the same:

$\left[4141,66,37 \cdot 5^{3}\right]$ with $\left(\langle 37,5+\sqrt{-974}\rangle \cdot P_{1}^{3}\right)^{2}=\langle 4141+66 \sqrt{-974}\rangle$, and

$\left[3167,108,37 \cdot 5^{3}\right]$ with $\left(\langle 37,32+\sqrt{-974}\rangle \cdot P_{1}^{3}\right)^{2}=\langle 3167-108 \sqrt{-974}\rangle$. Since

$$
\langle 4141+66 \sqrt{-974}\rangle \cdot\langle 14651+174 \sqrt{-974}\rangle=\langle 3167+108 \sqrt{-974}\rangle \cdot\left\langle 5^{6}\right\rangle,
$$

we have

$$
\left[4141,66,37 \cdot 5^{3}\right]+\left[14651,174,5^{6}\right]=\left[3167,108,37 \cdot 5^{3}\right],
$$

and hence according to our definition we have $\beta(37)=\left[3167,108,37 \cdot 5^{3}\right]$. 


\section{References}

[1] N. Baldisserri, The group of primitive quasi-Pythagorean triples, Rend. Circ. Mat. Palermo (2) 48(2) (1999), 299-308.

[2] D. Cox, Primes of the form $x^{2}+n y^{2}$ Fermat, Class Field Theory and Complex Multiplication, A Wiley-Interscience Publication, John Wiley \& Sons, Inc., New York, 1989.

[3] E. Eckert, The group of primitive Pythagorean triangles, Math. Mag. 57(1) (1984), 22-27.

[4] N. Krylov and L. Kulzer, The group of primitive almost Pythagorean triples, Involve 6(1) (2013), 13-24.

[5] F. Lemmermeyer, Higher Descent on Pell Conics III, The First 2-Descent, preprint 2003.

[6] D. Marcus, Number Fields, Universitext, Springer-Verlag, New York-Heidelberg, 1977.

[7] O. Taussky, Sums of squares, Amer. Math. Monthly 77 (1970), 805-830.

[8] P. Zanardo and U. Zannier, The group of Pythagorean triples in number fields, Ann. Mat. Pura Appl. (4)159 (1991), 81-88. 